\title{
Protective Effect of Stem Cells from Toxicity Induced by Gulf War Illness (GWI) Serum in N2A Neuroblastoma Cells
}

\author{
Effie-Photini C. Tsilibary ${ }^{1,2}$, Danielle Carlson ${ }^{1,2}$, Apostolos P. Georgopoulos ${ }^{1,2 *}$ \\ 'Brain Sciences Center, Department of Veterans Affairs Health Care System, Minneapolis, Minnesota, USA \\ ${ }^{2}$ Department of Neuroscience, University of Minnesota Medical School, Minneapolis, Minnesota, USA
}

\section{Article Info}

\section{Article Notes}

Received: June 14, 2021

Accepted: August 20, 2021

\section{${ }^{*}$ Correspondence:}

Dr. Apostolos P. Georgopoulos, Brain Sciences Center (11B), Minneapolis VAHCS, One Veterans Drive, Minneapolis, MN 55417, USA; Email: omega@umn.edu.

(c) 2021 Georgopoulos AP. This article is distributed under the terms of the Creative Commons Attribution 4.0 International License

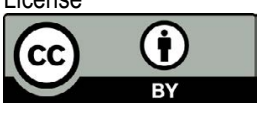

Keywords:

Gulf War Illness (GWI)

N2A cells

Apoptosis

PA63

Stem cells

\section{ABSTRACT}

Gulf War Illness (GWI) afflicted many veterans of the 1990-91 Gulf War with multiple symptoms worsening with time. The reasons for GWI have not been elucidated but may include toxicity due to inflammatory factors induced by vaccines administered to deployed and nondeployed veterans. In particular, the anthrax vaccine may have harmful effects in veterans lacking specific protective HLA alleles, as we reported previously, using a murine neuroblastoma N2A cell culture system. Lack of these protective alleles could allow several vaccine antigens to circulate chronically, resulting in protracted low-grade inflammation accompanying the disease. When N2A cells were exposed to GWI serum or the antigen of the anthrax vaccine, the cells underwent apoptosis due to compromised cell membrane, mitochondrial and cytoskeletal function. Elucidation of mechanisms of GWI should provide clues for therapy. Since antigen-induced inflammation accompanies GWI and stem cells were reported to have antimicrobial activity, we examined the effect of murine stem cells co-cultured with N2A cells before exposure to GWI serum and also Protective Antigen PA63, the main component of the anthrax vaccine. The presence of stem cells completely prevented GWI serum toxicity, since it resulted in inhibition of apoptosis. Moreover, cultures of stem cells exposed to PA63 resulted in the degradation of this antigen. We conclude that stem cells can protect against vaccine-induced toxic components of the GWI serum in N2A cells, prompting further studies on the possible beneficial effects of these cells in GWI.

\section{List of abbreviations}

Gulf War: GW; Gulf War Illness (GWI); Human Leucocyte Antigen system HLA; Fetal bovine serum FBS; sodium dodecyl sulfate: SDS; Mesenchymal Stem Cells: MSC; Terminal deoxynucleotidyl transferase mediated dUTP Nick End Labeling assay: TUNEL; neurocognitive mood symptoms: NCM; 4',6-diamidino-2-phenylindole stain: DAPI; Protective Anthrax Antigen 63: PA63; Mouse Neuroblastoma Cells: N2A cells OD: optical density

\section{Introduction}

GWI symptomatology appeared in about one third of deployed and nondeployed veterans of the Persian Gulf War with multiple symptoms, including neurocognitive and mood dysfunction ${ }^{1}$. Insofar as Central nervous system (CNS) involvement in GWI is concerned, we reported previously a number of functional and structural brain abnormalities in GWI, such as changes in synchronous neural communication patterns $^{2-5}$ and subcortical brain atrophy in certain GWI patients ${ }^{6}$. Moreover, active inflammation has been reported in GWI and, in addition, a positive association of the levels of C-reactive protein (CRP), an inflammatory marker, with GWI symptom severity? ${ }^{7}$ We have also reported that GWI patients lack six specific protective HLA 
alleles, and the severity of their disease correlates with the number of missing alleles, indicating compromised immunity $^{8}$. Given the impaired ability of these patients to respond to pathogens, it is possible that antigens present in the multiple vaccines administered to veterans could not be neutralized thus circulating for a prolonged time and resulting in chronic low-grade inflammation ${ }^{9,10}$. In order to assess the effects of inflammation, also affecting the CNS, we developed a murine neuroblastoma N2A cell culture system, in which we exposed neural cells to serum from GWI patients lacking all protective HLA alleles and observed remarkable toxic effects, including retraction of cell processes, cell aggregation and enhanced apoptosis, compared to healthy serum ${ }^{11}$. These effects were prevented to a large extent by the simultaneous presence of antibodies to PA63 in cells exposed to GWI serum, thus indicating the presence of this antigen in GWI patients ${ }^{12}$ Moreover, GWI serum toxicity was reproduced when N2A cells were exposed to protective antigen PA63 of the anthrax vaccine which was administered to veterans of the Gulf War suffering from GWI . The observed structural and functional changes induced by the presence PA63 in the culture medium included compromised cell membrane integrity as well as impaired mitochondrial and cytoskeletal function ${ }^{13}$. Hence, GWI adverse effects in neural cells could be due, at least in part, to the presence of the anthrax vaccine antigen PA63, which could contribute to the chronic pathogen-induced inflammation accompanying this disease.

Presently the treatment of GWI is symptomatic and there is no cure. Considering the inflammatory nature of the disease, new approaches should be considered as new means to intervene with symptoms and progression. In this respect, stem cells have been reported to combat pathogens with direct and indirect antimicrobial activity. More specifically, mesenchymal stem cells (MSC) exhibited spontaneous direct bactericidal activity in vitro by secreting soluble factors and acting synergistically with antibiotics, when administered in vivo to mice with established $\mathrm{S}$. aureus biofilm infections ${ }^{14}$. Factors secreted by MSC were found to include antimicrobial / host defense peptides and proteins (AMPs). In fact, MSCs have been reported to increase bacterial clearance in preclinical models of sepsis $^{15}$.

We therefore explored the in vitro effects of murine stem cells co-cultured with N2A cells, in order to assess whether these cells had protective effects against pathogen- and GWI-induced N2A cell toxicity. When GWI serum or PA63 were present in the culture medium of the mixed cultures or stem cell cultures (in the case of PA63), GWI seruminduced toxic effects leading to apoptosis were completely prevented, and PA63 was degraded.

\section{Materials and Methods}

\section{Serum}

We used serum from 15 GWI veterans with substantial GWI symptoms and serum from a healthy GW veteran; patients did not carry any of the $6 \mathrm{GWI}$-protective alleles ${ }^{8}$, whereas the control carried 2 such alleles. The study was approved by the relevant Institutional Review Board and informed consent for using their serum was obtained from all participants.

\section{Cell culture}

Neuro-2A neuroblastoma (N2A) cells were cultured in Eagle's minimal essential medium (EMEM, ATCC, VA, USA) containing 10\% fetal bovine serum (ThermoFisher Scientific, Waltham, MA) in poly-D-lysine coated, 8-chamber slides at a concentration of 50,000/well for 48 hours. The medium was then changed to Neurobasal containing N2 supplement and L-glutamine (ThermoFisher Scientific, Waltham, MA), in the absence (medium control) or presence of human serum (healthy or GWI). For all experiments, human serum was added in 2 combinations: control (10\%), and GWI (10\%).

\section{Stem cells}

Murine stem cells (ATCC Cat. No. CRL-11115) were cultured in medium containing 85\% DMEM, 15\% nonheat inactivated fetal bovine serum, and $0.1 \%$ beta mercaptoethanol (BME), with frequent medium changes until the cells were approximately $70 \%$ confluent. The cells were then released with trypsin and used further in mixed N2A-stem cell cultures as described below.

\section{Co-cultures of stem and $\mathrm{N} 2 \mathrm{~A}$ cells}

N2A cells seeded on 8-chamber slides at a concentration of 40,000 cells per chamber or 40,000 cells/chamber and 2,500 stem cells. Neurobasal medium was added in the N2A cells, and in 50\% Neurobasal, 50\% stem cell medium was added in the mixed cultures. 48 hours later, the N2A and mixed cell cultures were exposed to $10 \%$ GWI serum from $15 \mathrm{GWI}$ patients or healthy serum, and were allowed to incubate for 2 days before being processed for apoptosis.

\section{Stem cell cultures for exposure to PA63}

For exposure to PA63, 25,000 stem cells per well were seeded to 48-well plates and were kept in a stem cell medium for 48 hours. At the end of the incubation period 2-3 $\mu$ g PA63 (Creative Diagnostics, Shirley, NJ, USA) were added in the medium for $45 \mathrm{~min}$. Then equal amount of PA63 in medium in which PA63 was added and incubated with the co-cultures were used before and after incubation, for sodium dodecyl sulfate (SDS)-gel eletrophoresis and western blotting. 
Western blotting for detection of PA63 in the presence and absence of stem cells in $\mathrm{N} 2 \mathrm{~A}$ cell cultures

Equal amounts of medium containing $3 \mu \mathrm{g}$ of PA63 before exposure to N2A-stem cells and medium after incubation with the co-cultures were loaded on $4-12 \%$ gradient SDSPAGE gels for electrophoresis, and then transferred onto nitrocellulose membranes. Membranes were blocked with 5\% non-fat milk, $0.1 \%$ Tween-20 in TBS for 1 hour at room temperature, and incubated overnight at $4{ }^{\circ} \mathrm{C}$ with primary monoclonal (CABT-51076MA) or polyclonal antiserum (Cat. No. CPBT-66806RA; both from Creative Diagnostics, Shirley, NY, US) and/or polyclonal anti-anthrax PA63 antibodies (Creative Diagnostics, Shirley, NJ) in dilution 1:500 according to manufacturers' instructions. Membranes were washed in TBS/Tween-20 and incubated with horseradish peroxidase-conjugated secondary antirabbit and anti-mouse antibodies in dilution 1:30,000 (GE healthcare, Buckinghamshire, UK) and $5 \%$ bovine serum albumin (BSA) for 1 hour at room temperature. Blots were visualized with an ECL chemiluminescence substrate (ThermoFisher Scientific, Waltham, MA).

Quantification of protein levels was performed by densitometry of the immunoreactive bands using the Odyssey FC imaging system (Li-COR Biosciences, Lincoln, $\mathrm{NE}$.

Cell Apoptosis with Terminal deoxynucleotidyl transferase mediated dUTP Nick End Labeling assay (TUNEL) assay

The extent of cell apoptosis in cultures of Neuro-2A cells also mixed with stem cells was examined at 2 days postexposure to healthy and GWI serum from each of $15 \mathrm{GWI}$ patients, using 4- and 8-chamber glass slides (ThermoFisher Scientific, Waltham, MA) coated with poly-D-lysine at $50 \mu \mathrm{g} / \mathrm{ml}$ as mentioned above. N2A cells were seeded at a concentration of 50,000 cells per chamber, or 40,000 N2Acells and 10,000 stem cells, in $1 \mathrm{ml}$ of Neurobasal/ $\mathrm{N} 2 /$ L-glutamine medium for 2 days. In sequence, $10 \%$ of healthy or GWI serum, incubated for $60 \mathrm{~min}$ at $37^{\circ} \mathrm{C}$ was added for 2 more days. At the end of the incubation period, the cells were examined for apoptosis. Apoptotic cells were detected using the In Situ Cell Death Detection Kit, TMR red (Terminal deoxynucleotidyl transferase (TdT) enzyme and fluorochrome labeling solution) according to the manufacturer's protocol. Briefly, the cells were fixed in ice-cold methanol for $10 \mathrm{~min}$ at room temperature, rinsed with PBS and permeabilized with $0.1 \%$ Triton X-100 in PBS for 3 min on ice. The cells were then incubated with $150 \mu \mathrm{l}$ of TUNEL reaction mixture for $60 \mathrm{~min}$ at $37^{\circ} \mathrm{C}$ in the dark (In situ Cell Death Detection Kit, TMR red, (ThermoFisher scientific, Waltham MA), or Click-iT Alexa Fluor488 Assay (ThermoFisher scientific, Waltham MA). The cells were then washed $3 \mathrm{X}$ with PBS and Diamond AntiFade mounting medium with 4',6-diamidino-2-phenylindole (DAPI) stain (ThsermoFisher Scientific, Waltham, MA) was used for visualization of nuclei, using an Olympus 3000 confocal microscope. an Olympus 3000 confocal microscope. (ThermoFisher Scientific, Waltham, MA). Eight-10 images were obtained from different fields from a minimum of two experiments with each different experimental condition. Apoptosis was then calculated with ImageJ software by measuring the number of TUNEL-labeled cells (red nuclei) relative to the total cell number (DAPI-stained nuclei).

\section{Statistical analysis}

For statistics, the paired $t$ test was used in all instances using Microsoft Excel.

\section{Results and Discussion}

\section{Apoptotic assay}

Apoptotic nuclei were identified by red staining with the TUNEL marker indicating DNA breaks (Figure 1) as we reported previously. ${ }^{11,12}$ The presence of stem cells in N2A cultures exposed to each of the 15 GWI sera, prevented cell apoptosis which was similar to the extent observed in medium and the healthy controls (Figure 2).

\section{Discussion}

In GWI, a multi-symptom disease of veterans of the Gulf War (1990-1991) brain function is also affected, characterized by neurological, cognitive and mood-related (NCM) symptoms which are often accompanied by mental health disorders, predominantly posttraumatic stress disorder (PTSD) ${ }^{16}$. These symptoms accompany functional and structural brain abnormalities in GWI, such as changes


Figure 1: Control (blue, 4',6-diamidino-2-phenylindole stain staining of N2A cell nuclei indicates healthy cells. Red-stained, Terminal deoxynucleotidyl transferase mediated dUTP Nick End Labeling (TUNEL) nuclei (arrows) indicate DNA breaks in apoptotic cells. A, B: Control N2A cells; C, D N2A cells exposed to $10 \% \mathrm{GWI}$ serum for 2 days. A, C: N2A cells cultured in the absence of stem cells. B, D: N2A cells cultured in the presence of stem cells. 


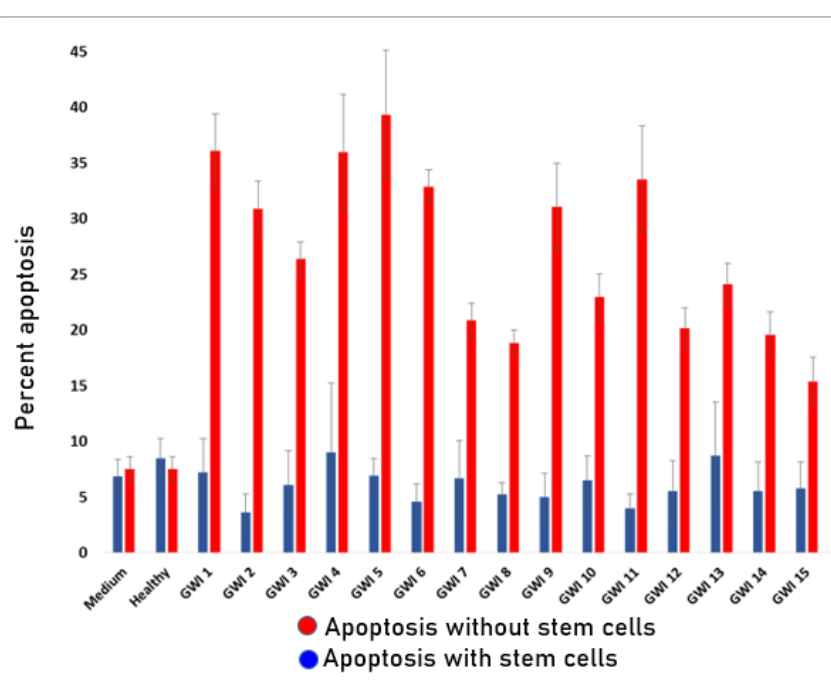

Figure 2: Extent of apoptosis in N2A cultures exposed to GWI serum in the absence and presence of stem cells in the culture. N2A cell apoptosis in each of the $15 \mathrm{GWI}$ sera was significantly enhanced (about 20-40\%) compared the medium and healthy controls (7$8 \% ; p<0.001)$. The simultaneous presence of stem cells with $\mathrm{N} 2 \mathrm{~A}$ cells prior to exposure to GWI totally prevented enhancement of apoptosis which was similar to the medium and healthy controls (approximately $3-8 \% ; p<0.001$ ).


stem cells for $45 \mathrm{~min}$, and stem cell medium before and after incubation was examined by western blotting (Figure 3A).

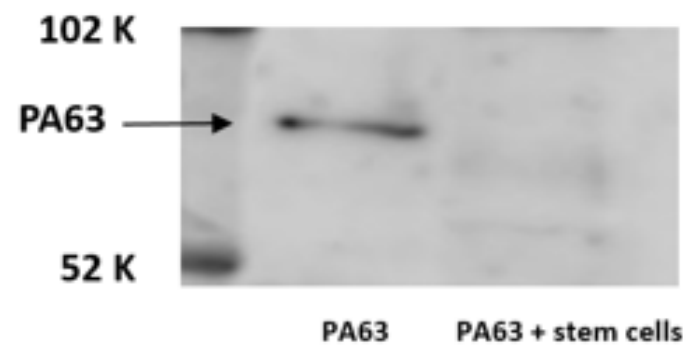

Figure 3A: Western blotting of PA63 before and after exposure to cocultures N2A and stem cells. Following exposure to the co-cultures PA63 was degraded by approximately forty percent. (Figure 3B).



Figure 3B: PA63 band OD before and after exposure to stem cells. $* p<0.05$ in synchronous neural communication patterns ${ }^{2-5}$ and subcortical brain atrophy in certain GWI patients ${ }^{6}$. Thus various neuropsychological deficits, particularly in the domains of attention, executive functioning, memory, and motor functioning, among other, were reported ${ }^{17}$. Overall then, GWI should be included in diseases in which neurodegeneration occurs.

In order to better examine possible toxic effects of GWI in the brain, we have used extensively an in vitro neural cell system, in which N2A mouse neuroblastoma cells were exposed to serum from GWI patients, and examined the harmful effects of serum from this disease, which leads to structural and functional cell changes, resulting in cell apoptosis ${ }^{11-13}$. These in vitro data agree with neuronal cell loss in vivo, which was indicated by brain atrophy in specific brain areas in certain GWI patients ${ }^{6}$. It follows that replacement of the lost cells would be beneficial for these patients. In order to assess this possibility, we used murine stem cells co-cultured with N2A cells in our in vitro cell system, in order to test a possible effect upon exposure to GWI serum.

Presently there is no cure for brain or other symptoms of GWI besides symptomatic treatment. Since brain symptoms then prevail in this disease, the development of new means to interfere with NCM symptoms is pivotal. In recent years, stem cell therapy has been intensely investigated as an alternative treatment using cell therapy. In particular stem cells including mesenchymal stem cells (MSC) are pluripotent stem cells with ability for selfrenewal and multidirectional differentiation ${ }^{18}$. Thus MSC may be considered a reliable source of neural cells for replacement cell therapy in diseases such as GWI, given the observed subcortical brain atrophy in certain GWI patients ${ }^{6}$, which indicates neuronal cell loss.

Neuronal cell loss in our in vitro system was indicated to be due to inflammatory factors at least in part. These factors could include components of pathogens present in the vaccines administered to $\mathrm{GW}$ veterans who had impaired immunity due to the lack of specific protective HLA alleles ${ }^{8}$. Evidence about the persisting existence of vaccine-derived pathogens was obtained in experiments in which human IgG co-incubated with GWI serum resulted in substantial protection from cell apoptosis ${ }^{19}$. Moreover, the presence of antibodies against the anthrax vaccine which has toxic effects, also prevented GWI-induced toxic effects and N2A cell apoptosis ${ }^{12}$. It is therefore possible that part of the adverse effects of GWI in the brain could involve the protracted presence of vaccine-derived pathogens.

Stem cells could then convey additional beneficial effects since mesenchymal stem cells (MSC) were reported to exhibit spontaneous direct bactericidal activity in vitro by secreting soluble factors and acting synergistically with antibiotics, when administered in vivo to mice with 
established S. aureus biofilm infections ${ }^{14}$. Factors secreted by MSC were found to include antimicrobial / host defense peptides and proteins (AMPs). In fact, MSCs have been reported to increase bacterial clearance in preclinical models of sepsis ${ }^{15}$. Apparently then, the use of stem cells should have several protective effects in GWI.

We tested the effect of stem cells by establishing mixed cell cultures containing both $\mathrm{N} 2 \mathrm{~A}$ and stem cells, and exposing these co-cultures to GWI and also stem cells to PA63 which is derived from the anthrax vaccine after its administration ${ }^{13}$. The presence of stem cells totally prevented cell apoptosis, which was observed to be similar to the medium and healthy controls. Moreover, administration of PA63 antigen to stem cell cultures resulted in partial degradation of this antigen within 45 min, confirming the previously reported stem cell antimicrobial/host defense peptides and proteins as protective factors secreted from these cells ${ }^{14,15}$. These data indicate that the presence of stem cells had a strong protective effect from GWI and pathogen-induced cell damage in our in vitro system. If stem cells have similar effects in vivo, they could prevent neural cell apoptosis and should help preserve neuronal cells, in addition to promoting the formation of neurons newly differentiated from mesenchymal and other stem cells. These cells would also help for removing possible vaccine-induced pathogens and the resulting protracted inflammation which accompanies $\mathrm{GWI}^{7}$. It is therefore worthwhile to consider further exploration regarding the use of stem cells as new and effective means to interfere with GWI disease and its progression. It is known that stem cells can successfully home to their target in the brain and initiate repair, making them a promising approach in cellular therapy in neurodegenerative diseases including $\mathrm{GWI}^{20}$. Possible sources of self- derived MSC in adult subjects include the bone marrow and fat tissue ${ }^{18}$; Stem cells from these sources could be used in order to establish their protective effect in vivo in GWI.

\section{Conclusions}

The data obtained in this series of experiments strongly suggest a pivotal protective role of stem cells against the in vitro harmful effects of GWI serum in a model system of N2A murine neuroblastoma cells, by inhibiting GWI seruminduced apoptosis and also degrading the harmful PA63 anthrax antigen. Possible in vivo protection in GWI patients remains to be substantiated.

\section{Authors' contributions}

Conceptualization: A.P.G. and E.-P.C.T.; data curation:

E.-P.C.T., D.C ; formal analysis: E.-P.C.T. and A.P.G.; investigation: E.P.C. and D.C; methodology: E.-P.C.T., D.C. and A.P.G.; project administration: E.-P.C.T; visualization: E.-P.C.T.; writing-original draft: E.-P.C.T.; writing-review and editing:
E.-P.C.T., D.C. and A.P.G. All authors have read and agreed to the published version of the manuscript.

\section{Acknowledgements}

This work was partially supported by the American Legion of Minnesota BStem cell Fund, the University of Minnesota American Legion Brain Sciences Chair, and the U.S. Department of Veterans Affairs. The sponsors had no role in the current study design, analysis or interpretation, or in the writing of this paper. The contents do not represent the views of the U.S. Department of Veterans Affairs or the United States Government.

\section{References}

1. Fukuda K, Nisenbaum R, Stewart G, et al. Chronic multisymptom illness affecting Air Force veterans of the Gulf War. JAMA. 1998;280:981-988

2. Engdahl BE, James LM, Miller RD, et al. A magnetoencephalographic (MEG) study of Gulf War Illness (GWI). EBioMedicine. 2016;12:127132.

3. James LM, Engdahl BE, Leuthold AC, et al. Brain correlates of Human Leukocyte Antigen (HLA) protection in Gulf War Illness (GWI). EBioMedicine. 2016;13:72-79.

4. Georgopoulos AP, James LM, Carpenter AF, et al. Gulf War illness (GWI) as a neuroimmune disease. Exp Brain Res. 2017;235:32173225. 8. Engdahl BE, James LM, Miller RD, et al. Brain function in Gulf War Illness (GWI) and associated mental health comorbidities. J Neurol Neuromedicine. 2018;3:24-34.

5. Engdahl BE, James LM, Miller RD, et al. Brain function in Gulf War Illness (GWI) and associated mental health comorbidities. J Neurol Neuromedicine. 2018;3:24-34.

6. Christova P, James LM, Engdahl BE, et al. Subcortical brain atrophy in Gulf War Illness. Exp Brain Res. 2017;235:2777-2786.

7. James LA, Engdahl BE, Johnson RA, et al. Gulf War Illness and Inflammation: Association of symptom severity with C-reactive protein. J Neurol Neuromed. 2019; 4(2): 15-19.

8. Georgopoulos AP, James LM, Mahan MY, et al. Reduced Human Leukocyte Antigen (HLA) protection in Gulf War Illness (GWI). EBioMedicine. 2016; 3: 79-85.

9. Tamburini BA, Burchill MA, Kedl RM. Antigen capture and archiving by lymphatic endothelial cells following vaccination or viral infection. Nat Commun. 2014;5:3989. doi:10.1038/ncomms4989.

10. Tew JG, Mandel TE. Prolonged antigen half-life in the lymphoid follicles of specifically immunized mice. Immunol. 1979;37:69-76.

11. Georgopoulos AP, Tsilibary EC, Souto EP, et al. Adverse effects of Gulf War Illness (GWI) serum on neural cultures and their prevention by healthy serum. J Neurol Neuromedicine. 2018;3(2):19-27.

12. Tsilibary EC, Souto EP, Kratzke M, et al. Anthrax and Gulf War Illness: Evidence for the Presence of Harmful Anthrax Antigen PA63 In the Serum of Veterans with GWI. Journal of Neurol Neuromedicine. 2019;4(6):1-9.

13. Tsilibary EC, Souto EP, Kratzke M, et al. Anthrax Protective Antigen 63(PA63): Toxic Effects in Neural Cultures and Role in Gulf War Illness (GWI). Neurosci Insights. 2020;15:2633105520931966. doi:10.1177/2633105520931966

14. Chow L, Johnson V, Impastato R, et al. Antibacterial activity of human mesenchymal stem cells mediated directly by constitutively secreted factors and indirectly by activation of innate immune effector cells. Stem Cells Transl Med. 2020 Feb;9(2):235-249. 
15. Alcayaga-Miranda F, Cuenca J, Khoury M. Antimicrobial Activity of Mesenchymal Stem Cells: Current Status and New Perspectives of Antimicrobial Peptide-Based Therapies. Front Immunol. 2017;8:339. doi: 10.3389/fimmu.2017.00339. PMID: 28424688; PMCID: PMC5371613.

16. Engdahl BE, James LM, Miller RD, et al. Brain Function in Gulf War Illness (GWI) and Associated Mental Health Comorbidities. J Neurol Neuromedicine. 2018;3(4):24-34. PMID: 30882065; PMCID: PMC6417922.

17. Jeffrey MG, Krengel M, Kibler JL, et al. Neuropsychological Findings in Gulf War Illness: A Review. Front Psychol. 2019 Sep 26;10:2088. doi: 10.3389/fpsyg.2019.02088. PMID: 31616335; PMCID: PMC6775202.
18. Yao P, Zhou L, Zhu L, et al. Mesenchymal Stem Cells: A Potential Therapeutic Strategy for Neurodegenerative Diseases. Eur Neurol. 2020;83(3):235-241. doi: 10.1159/000509268. Epub 2020 Jul 20. PMID: 32690856.

19. Tsilibary EPC, Souto EP, James LM, et al. Human Immunoglobulin G (IgG) neutralizes adverse effects of Gulf War Illness (GWI) serum in neural cultures: Paving the way to immunotherapy for GWI. J Neurol Neuromed. 2018;3(5):23-28

20. Chia YC, Anjum CE, Yee HR, et al. Stem Cell Therapy for Neurodegenerative Diseases: How Do Stem Cells Bypass the BloodBrain Barrier and Home to the Brain? Stem Cells Int. 2020 Sep 4;2020:8889061. doi: 10.1155/2020/8889061. PMID: 32952573; PMCID: PMC7487096. 